\title{
Degenerate Frobenius manifolds and the bi-Hamiltonian structure of rational Lax equations
}

\author{
I.A.B. Strachan \\ Department of Mathematics, University of Hull, \\ Hull, HU6 7RX, England. \\ e-mail: i.a.b.strachan@hull.ac.uk
}

\begin{abstract}
The bi-Hamiltonian structure of certain multi-component integrable systems, generalizations of the dispersionless Toda hierarchy, is studied for systems derived from a rational Lax function. One consequence of having a rational rather than a polynomial Lax functions is that the corresponding bi-Hamiltonian structures are degenerate, i.e. the metric which defines the Hamiltonian structure has vanishing determinant. Frobenius manifolds provide a natural setting in which to study the bi-Hamiltonian structure of certain classes of hydrodynamic systems. Some ideas on how this structure may be extended to include degenerate bi-Hamiltonian structures, such as those given in the first part of the paper, is given.
\end{abstract}

\section{Introduction}

Poisson brackets of hydrodynamic type were introduced by Dubrovin and Novikov in [1] where they gave a complete description of Poisson brackets of the form

$$
\left\{u^{i}(x), u^{j}(y)\right\}=g^{i j}[u(x)] \delta^{\prime}(x-y)+\Gamma_{k}^{i j}[u(x)] u^{k}(x) \delta(x-y)
$$

under the non-degenerate condition $\operatorname{det}\left(g^{i j}\right) \neq 0$. This defines a skew-symmetric Poisson bracket on functionals

$$
\{I, J\}=\int d x \frac{\delta I}{\delta u^{i}(x)} \widehat{A^{i j}} \frac{\delta J}{\delta u^{j}(x)}
$$

where

$$
\widehat{A^{i j}}=g^{i j}[u(x)] \frac{d}{d x}+\Gamma_{k}^{i j}[u(x)] u^{k}(x)
$$


The conditions on $g^{i j}$ and $\Gamma_{k}^{i j}$ necessary in order for (1) to define a Hamiltonian structure, under the non-degenerate condition $\operatorname{det}\left(g^{i j}\right) \neq 0$, have a natural geometric interpretation |11:

Theorem 1 Under the non-degenerate condition $\operatorname{det}\left(g^{i j}\right) \neq 0$ the bracket (1) defines a Hamiltonian structure if and only if:

a) $\mathbf{g}=\left(g^{i j}\right)^{-1}$ defines a (pseudo)-Riemannian metric;

b) $\Gamma^{i j}{ }_{k}=-g^{i s} \Gamma_{s k}^{j}$, where $\Gamma_{s k}^{j}$ are the Christoffel symbols of the Riemannian connection defined by $\mathbf{g}$;

c) the Riemann curvature tensor of $\mathbf{g}$ vanishes.

This result, and its interpretation in terms of differential geometry, rests on the nondegeneracy condition on the metric. However, this is not a necessary condition for (11) to define a Hamiltonian structure and the full result, with no a priori restriction on $g^{i j}$ was derived by Grinberg [2] and Dorfmann [3]:

Theorem 2 The bracket (1) defines a Hamiltonian structure if and only if the pair $(g, \Gamma)$ satisfy the conditions:

$$
\begin{aligned}
g^{i j} & =g^{j i} ; \\
\frac{\partial g^{i j}}{\partial u^{k}} & =\Gamma_{k}^{i j}+\Gamma_{k}^{j i} ; \\
g^{i j} \Gamma_{i}^{r s} & =g^{r i} \Gamma_{i}^{j s} ; \\
\Gamma_{t}^{i j} \Gamma_{r}^{t k}-\Gamma_{t}^{i k} \Gamma_{r}^{t j} & =g^{t i}\left(\frac{\partial \Gamma_{r}^{j k}}{\partial u^{t}}-\frac{\partial \Gamma_{t}^{j k}}{\partial u^{r}}\right)
\end{aligned}
$$

and

$$
\sum_{\text {cyclic sum on } i, j, k}\left[\left(\frac{\partial \Gamma_{t}^{i j}}{\partial u^{q}}-\frac{\Gamma_{q}^{i j}}{\partial u^{t}}\right) \Gamma_{r}^{t k}+\left(\frac{\partial \Gamma_{t}^{i j}}{\partial u^{r}}-\frac{\Gamma_{r}^{i j}}{\partial u^{t}}\right) \Gamma_{q}^{t k}\right]=0 .
$$

If $\operatorname{det} g^{i j} \neq 0$ then the last equation is a consequence of the earlier equations.

[N.B. there is a minor error in [2] in the order of the indices in equation (4)]. In this more general situation it is not possible to give a clear geometric interpretation of these equations. They define an integrable distribution but their differential geometric content is less clear. One can define a covariant derivative like object

$$
\nabla^{i} \xi^{j}=\partial^{i} \xi^{j}-\Gamma_{k}^{i j} \xi^{k}
$$


where $\partial^{i}=g^{i j} \partial_{j}$, with the property (when suitably extended to tensors) that $\nabla^{i} g^{j k}=0$, though the 'connection' cannot defined in terms of the 'metric'. With such a covariant derivative one can introduce a 'curvature' by the equation

$$
\left(\nabla^{r} \nabla^{s}-\nabla^{s} \nabla^{r}\right) \xi^{t}=-R_{k}^{r s t} \xi^{k}
$$

and the third equation above is now just the vanishing of this curvature. Such a description is not very natural; one cannot lower indices and the interpretation of the last equation remains unclear. However the terms 'metric' and 'connection' will be used to denote these objects, and a pair satisfying these equations will be called a $(g, \Gamma)$-pair.

The purpose of this paper is to study the bi-Hamiltonian structure of dispersionless integrable systems defined by the Lax equation 1

$$
\frac{\partial \mathcal{L}}{\partial \tau_{n}}=\left\{\left(\mathcal{L}^{\frac{n}{N-M}}\right)_{+}, \mathcal{L}\right\}_{P B}
$$

where $\{f, g\}_{P B}=p\left(\partial_{p} f \partial_{x} g-\partial_{x} f \partial_{p} g\right), \mathcal{L}$ is given by a rational function

$$
\mathcal{L}=\frac{\text { polynomial of degree } \mathrm{N}}{\text { polynomial of degree } \mathrm{M}}
$$

with the single constraint $N>M$, and ()$_{+}$denotes the projection onto non-negative powers of $p$ under a formal expansion in powers of $p$. In an earlier paper [4 this system was studied but a complete description of the Hamiltonian structure was not given. The simplest example of such a system is the continuum Toda equations

$$
\begin{aligned}
& S_{\tau}=P_{x}, \\
& P_{\tau}=P S_{x} .
\end{aligned}
$$

which is generated for the above Lax equation (7) with a Lax function

$$
\mathcal{L}=p^{2}+S(x, t)+\frac{P(x, t)}{p} .
$$

This paper aims to extend these earlier results from polynomial Lax functions to rational Lax functions and to relate these results to the theory of Frobenius manifolds [5]. It will turn out that in the rational case the Hamiltonian structure is degenerate, so the more general description of Grinberg and Dorfmann will have to be utilised to give a complete description of the bi-Hamiltonian structure of the hierarchy. This in turn implies that a new concept of a degenerate Frobenius manifold is required.

In the next section a summary of the pertinent result of [4] will be given, and this will also serve to fix the notation used. Full details will not be given and the reader should

\footnotetext{
${ }^{1}$ The variables $\tau_{n}$ will be used to denote the times, $t$ being reserved for flat coordinates in which the components $\eta^{i j}$ are constants.
} 
consult the earlier paper for the proofs. In section 3 the polynomial case will be studied in more detail (and this will relate the results of 4 to more recent work of Dubrovin and Zhang [6]) before the full rational case is studied in section 4 . The properties of a degenerate Frobenius manifold is introduced by way of an extended example in section 5 .

Throughout this paper various different coordinate systems will be used, and the resulting transformations from one system to another will be important. The notation $g^{i j}(s)$ will be used to denote the components of the metric in the $s^{i}$-coordinate system, so the transformation from $s^{i}$ to $t^{i}$ coordinates will be written

$$
g^{i j}(t)=\frac{\partial t^{i}}{\partial s^{p}} \frac{\partial t^{j}}{\partial s^{q}} g^{p q}(s)
$$

rather than using different founts and alphabets for the different coordinate systems.

\section{Conservation Laws and Evolution Equations}

In order to study rational functions it is convenient, and indeed necessary in order to obtain some results, to factorize the numerator and denominator of the rational function, so

$$
\begin{aligned}
\mathcal{L} & =\frac{\prod_{i=1}^{N}\left(p+u^{i}\right)}{\prod_{i=N+1}^{N+M}\left(p+u^{i}\right)} \\
& =\prod_{i=1}^{N+M}\left(p+u^{i}\right)^{\varepsilon_{i}}
\end{aligned}
$$

Here it will be assumed that $\varepsilon_{i}= \pm 1$ and that the numerator and denominator have no common root. With these conditions and $N>M$ the Lax function is of the general form

$$
\mathcal{L}=\text { polynomial of degree }(N-M)+\sum_{i=N+1}^{N+M} \text { simple poles } .
$$

Such a factorization of the Lax function was introduced by Kupershmidt [7] (though this could also be viewed as a Vieté transformation) and the variables $u^{i}$ will be called modified variables. One advantage of such a factorization is that it puts all the fields on an egalitarian footing, i.e. the permutation group $S^{N}$ acts on the zeros of $\mathcal{L}$ and the permutation group $S^{M}$ acts on the roots of $\mathcal{L}$, and this drastically reduces the complexity of the calculations.

The flows are given by the Lax equation (7) which may be calculated explicitly

$$
u_{\tau_{n}}^{i}=A_{i}^{(n)} u_{x}^{i}+\sum_{j \neq i} u^{i} B_{i j}^{(n)} u_{x}^{j}
$$

where 


$$
A_{i}^{(n)}=\left(\frac{\epsilon_{i} n}{N-M}-1\right) \sum_{\left\{r_{j}: \sum_{j=1}^{N+M} r_{j}=n\right\}}\left[\prod_{\substack{k=1 \\
k \neq i}}^{N+M}\left(\begin{array}{c}
\frac{\epsilon_{k} n}{N-M} \\
r_{k}
\end{array}\right)\left(u^{k}\right)^{r_{k}}\right]\left(\begin{array}{c}
\frac{\epsilon_{i} n}{N-M}-2 \\
r_{i}-1
\end{array}\right)\left(u^{i}\right)^{r_{i}}
$$

and $B_{i j}^{(n)}=$

$$
\frac{\epsilon_{j} n}{N-M} \sum_{\left\{r_{j}: \sum_{j=1}^{N+M} r_{j}=n-1\right\}}\left[\prod_{\substack{k=1 \\
k \neq i, j}}^{N+M}\left(\begin{array}{c}
\frac{\epsilon_{k} n}{N-M} \\
r_{k}
\end{array}\right)\left(u^{k}\right)^{r_{k}}\right]\left(\begin{array}{c}
\frac{\epsilon_{i} n}{N-M}-1 \\
r_{i}
\end{array}\right)\left(u^{i}\right)^{r_{i}}\left(\begin{array}{c}
\frac{\epsilon_{j} n}{N-M}-1 \\
r_{j}
\end{array}\right)\left(u^{j}\right)^{r_{j}} .
$$

Care has to be taken in evaluating the binomial coefficients for negative and fractional numbers. These must be interpreted in terms of $\Gamma$-function, so

$$
\left(\begin{array}{l}
a \\
b
\end{array}\right)=\frac{\Gamma(a+1)}{\Gamma(a-b+1) \Gamma(b+1)} .
$$

It also follows from the proof of these results (though not explicitly mentioned in [4]) that

$$
\begin{aligned}
\mathcal{C} & =\left.\mathcal{L}\right|_{p=0}, \\
& =\prod_{i=1}^{M+N}\left(u^{i}\right)^{\epsilon_{i}}
\end{aligned}
$$

is a independent of all the times, i.e.

$$
\frac{\partial \mathcal{C}}{\partial \tau_{n}}=0 \quad n=1, \ldots, \infty .
$$

The functions $\mathcal{C}$ will turn out to be a Casimir for the bi-Hamiltonian structure of this hierarchy.

Conservation laws are similarly defined, the conserved charges being given by

$$
Q^{(n)}=\frac{1}{2 \pi i} \oint \mathcal{L}^{\frac{n}{N-M}} \frac{d p}{p}
$$

These may be derived explicitly

$$
Q^{(n)}=\sum_{\left\{r_{i}: \sum_{i=1}^{N+M}\right.}\left\{\prod_{\left.r_{i}=n\right\}}^{N+M}\left(\begin{array}{c}
\frac{\epsilon_{i} n}{N-M} \\
r_{i}
\end{array}\right)\left(u^{i}\right)^{r_{i}}\right\}
$$

Under a suitable change of variable, these polynomials take the form of generalised hypergeometric functions, a result which remains to be exploited. The corresponding functionals

$$
H^{(n)}=\int Q^{(n)} d x
$$

will turn out to be the Hamiltonians of the system (9). 


\section{Polynomial Lax equations}

In this section the bi-Hamiltonian structure of the hierarchy defined by a Lax function

$$
\mathcal{L}=p^{-M} \prod_{i=1}^{N}\left(p+u_{i}\right), \quad 0<M<N
$$

will be derived, this generalising the results of [4] where the special case $M=1$ was studied. Having derived one Hamiltonian structure, the intersection form in the language of Frobenius manifold, one may use a result of Dubrovin to find a second compatible Hamiltonian structure.

Proposition 3 The Hamiltonian structure of the hierarchy defined by equation (1) is given by the non-degenerate metric

$$
g^{i j}(u)= \begin{cases}{[1-(N-M)] u^{i} u^{i}} & \text { if } i=j \\ u^{i} u^{j} & \text { if } i \neq j .\end{cases}
$$

Comment This is clearly a flat, non-degenerate metric, and so defines a Hamiltonian structure. What is less clear is whether this structure, coupled to the Hamiltonians given by (11), gives rise to the flows defined by (7). This may be shown to be the case by direct calculation. An alternative proof, viewing the polynomial as a reduction of the rational case, will follow from the Theorem 7 in section 4 .

A bi-Hamiltonian structure is more than just two Hamiltonian structures; the two structures $\{,\}_{1}$ and $\{,\}_{2}$ have to be compatible, i.e. $\{\}=,\{,\}_{1}+\lambda\{,\}_{2}$ must be a Hamiltonian structure for all values of $\lambda$. For non-degenerate Poisson brackets of hydrodynamic type this compatibility condition implies that, for arbitrary $\lambda$ :

(a) the metric $g^{i j}=g_{1}^{i j}+\lambda g_{2}^{i j}$ is flat (such a metric is sometimes referred to as a flat pencil);

(b) the metric connection for this metric has the form $\Gamma_{k}^{i j}=\Gamma_{1 k}^{i j}+\lambda \Gamma_{2 k}^{i j}$.

A result of Dubrovin [5] (actually a special case of a more general result of Magri [8]) will enable the bi-Hamiltonian structure to be found:

Lemma 4 If for a flat metric in some coordinate system $x^{1}, \ldots, x^{n}$ both the components $g^{i j}(x)$ of the metric and $\Gamma_{k}^{i j}(x)$ of the corresponding metric connection depend linearly on the coordinate $x^{\bullet}$ then the metrics

$$
\begin{aligned}
& g_{1}^{i j}=g^{i j} \\
& g_{2}^{i j}=\partial_{\bullet} g^{i j}
\end{aligned}
$$


form a flat pencil, under the assumption that $\operatorname{det}\left[g_{2}^{i j}\right] \neq 0$. The corresponding metric connection has the form

$$
\begin{aligned}
& \Gamma_{1 k}^{i j}=\Gamma_{k}^{i j}, \\
& \Gamma_{2 k}^{i j}=\partial_{\bullet} \Gamma_{k}^{i j} .
\end{aligned}
$$

The proof of this result is straightforward, and an alternative proof to that given in [5] will follow from a result given in the next section where this lemma is extended to degenerate Hamiltonian structures.

In order to find such a coordinate system it is necessary to perform a number of coordinate transformations on the metric (12). This will be achieved in two stages. First define variables [6]

$$
\begin{aligned}
z^{1} & =+x^{1}, \\
z^{i} & =+x^{i}-x^{i-1}, \quad i=1, \ldots, N-1, \\
z^{N} & =-x^{N-1}
\end{aligned}
$$

(so $\sum_{i=1}^{N} z^{i}=0$ ) and then

$$
u^{i}=e^{\frac{1}{N} x^{N}-z^{i}}, \quad i=1, \ldots, N .
$$

Such a coordinate transformation has a nature interpretation in terms of the Weyl group $W\left(A_{N-1}\right)$, which act by permutation of the coordinates $z^{i}$ on the hyperplane $\sum_{i=1}^{N} z^{i}=0$. In this $x$-coordinate systems the components of the metric become, up to an overall factor of $(M-N)$ :

$$
\begin{aligned}
& g^{i j}(x)=\left(\begin{array}{ccccc|c}
2 & -1 & 0 & \ldots & 0 & 0 \\
-1 & 2 & -1 & \ldots & 0 & 0 \\
0 & -1 & 2 & \ldots & 0 & 0 \\
\vdots & \vdots & \vdots & & \vdots & \vdots \\
0 & 0 & 0 & \ldots & 2 & 0 \\
\hline 0 & 0 & 0 & \ldots & 0 & -\frac{M}{N(N-M)}
\end{array}\right) \\
& =\left(\begin{array}{c|c}
\begin{array}{c}
\text { Cartan matrix } \\
\text { of } A_{N-1}
\end{array} & 0 \\
\hline 0 & -d_{M}^{-1}
\end{array}\right) .
\end{aligned}
$$

The final entry is defined naturally using the Weyl group structure on $A_{N-1}$,

$$
\begin{aligned}
d_{M} & =\frac{M(N-M)}{N}, \\
& =\left(\omega_{M}, \omega_{M}\right)
\end{aligned}
$$


where $($,$) is the Euclidean inner product and \omega_{i}$ are the fundamental weights [6].

What these coordinate transformation show is that the Hamiltonian structure coincides with those found by Dubrovin and Zhang, so their results may be used to complete the second part of this arguments. In particular they show that in terms of the symmetric functions

$$
\begin{gathered}
s^{1}=\sum_{i} u^{i}, \\
s^{2}=\sum_{i<j} u^{i} u^{j}, \\
\vdots \\
\vdots \\
s^{N}=\prod_{i} u^{i} .
\end{gathered}
$$

the metric (12) will be linear in the variable $s^{M}$, and hence lemma 4 may be used to find the bi-Hamiltonian structure. The Jacobian of this transformation from modified to the original variables is just the Vandemonde determinant,

$$
\begin{aligned}
\frac{\partial\left(s^{1}, \ldots, s^{N}\right)}{\partial\left(u^{1}, \ldots, u^{N}\right)} & =\left|\begin{array}{cccc}
1 & 1 & \ldots & 1 \\
\sum_{i \neq 1} u^{i} & \sum_{i \neq 2} u^{i} & \ldots & \sum_{i \neq N} u^{i} \\
\vdots & \vdots & \ddots & \vdots \\
\prod_{i \neq 1} u^{i} & \prod_{i \neq 2} u^{i} & \ldots & \prod_{i \neq N} u^{i}
\end{array}\right| \\
& =\prod_{i<j}\left(u^{i}-u^{j}\right) .
\end{aligned}
$$

This defines the discriminant hypersurface, a caustic, on which $\mathcal{L}$ has multiple roots. By assumption $\varepsilon_{i}= \pm 1$ so all the roots are simple and hence the fields are well defined away from this surface. Hence [6]:

Lemma 5 The metric (19), when written in terms of the symmetric variables $s^{i}$ is linear in the variable $s^{M}$.

Before performing these calculations one should note that in terms of these symmetric variables the Lax function takes the more familiar form

$$
\mathcal{L}=p^{-M}\left[p^{N}+p^{N-1} s^{1}+\ldots+s^{N}\right]
$$

these symmetric variables coinciding with the original, unmodified variables. It also follows from the Lax equation (7) that the variable $s^{M}$ is special for another reason, namely it is the single variable for which the conserved charges $Q^{(n)}$ obey the relation 


$$
Q^{(n-1)}=\text { constant } \frac{\partial Q^{(n)}}{\partial s^{M}}
$$

Proposition 6 The first Hamiltonian structure, in terms of the modified variables, is given by

$$
\begin{aligned}
\eta^{i j}(u) & =\mathcal{L}_{\frac{\partial}{\partial s^{\bullet}}} g^{i j}(u), \\
& =\frac{\partial}{\partial s^{\bullet}} g^{i j}-\frac{\partial \alpha_{\bullet}^{i}}{\partial u^{k}} g^{k j}-\frac{\partial \alpha_{\bullet}^{j}}{\partial u^{k}} g^{i k}
\end{aligned}
$$

where the functions $\alpha_{\bullet}^{i}(u)$ are defined by

$$
\frac{\partial}{\partial s^{\bullet}}=\alpha_{\bullet}^{i}(u) \frac{\partial}{\partial u^{i}}
$$

and $\mathcal{L}_{\frac{\partial}{\partial s^{\bullet}}}$ is the Lie derivative along the vector field $\frac{\partial}{\partial s^{\bullet}}$.

Proof The transformation between the modified variables and the symmetric variables induces the transformation

$$
\left(\begin{array}{c}
\frac{\partial}{\partial u^{1}} \\
\vdots \\
\frac{\partial}{\partial u^{N}}
\end{array}\right)=\left(\begin{array}{cccc}
1 & \sum_{j \neq 1} u^{j} & \ldots & \prod_{j \neq 1} u^{j} \\
\vdots & \vdots & \ddots & \vdots \\
1 & \sum_{j \neq N} u^{j} & \ldots & \prod_{j \neq N} u^{j}
\end{array}\right)\left(\begin{array}{c}
\frac{\partial}{\partial s^{1}} \\
\vdots \\
\frac{\partial}{\partial s^{N}}
\end{array}\right)
$$

and hence by inverting the Vandemonde determinant

$$
\frac{\partial}{\partial s^{\bullet}}=\alpha_{\bullet}^{i}(u) \frac{\partial}{\partial u^{i}},
$$

this defining the functions $\alpha_{\bullet}^{i}(u)$. By lemmas 4 and 5 it follows, by starting with the metric (12) in the $u^{i}$-variables, transforming to the $s^{i}$-variables, differentiating with respect to $s^{\bullet}$ and then transforming back to the $u^{i}$-variables that

$$
\eta^{i j}(u)=\frac{\partial u^{i}}{\partial s^{m}} \frac{\partial u^{j}}{\partial s^{n}} \frac{\partial}{\partial s^{\bullet}}\left[\frac{\partial s^{m}}{\partial u^{r}} \frac{\partial s^{n}}{\partial u^{s}} g^{r s}(u)\right]
$$

is the required flat metric, which defines the second Hamiltonian structure. Expanding yields

$$
\eta^{i j}(u)=\frac{\partial}{\partial s^{\bullet}} g^{i j}+\frac{\partial u^{i}}{\partial s^{m}}\left(\frac{\partial}{\partial s^{\bullet}} \frac{\partial s^{m}}{\partial u^{r}}\right) g^{r j}+\frac{\partial u^{j}}{\partial s^{n}}\left(\frac{\partial}{\partial s^{\bullet}} \frac{\partial s^{n}}{\partial u^{s}}\right) g^{i s} .
$$


But

$$
\begin{aligned}
{\left[\frac{\partial}{\partial s^{\bullet}}, \frac{\partial}{\partial u^{k}}\right] } & =\left[\alpha_{\bullet}^{i} \frac{\partial}{\partial u^{i}}, \frac{\partial}{\partial u^{k}}\right] \\
& =-\frac{\partial \alpha_{\bullet}^{j}}{\partial u^{k}} \frac{\partial}{\partial u^{j}} .
\end{aligned}
$$

This, together with the definition of the Lie derivative and

$$
\frac{\partial s^{\beta}}{\partial s^{\bullet}}=\delta_{\bullet}^{\beta}
$$

yields the result.

This proposition is just an application of Magri's more general result [8] .

Example 1 For arbitrary $N$ and $M=1$ the distinguished coordinate is $s^{N}($ so $\bullet=N$ in the above formulae) and the functions $\alpha_{N}^{i}$ are

$$
\alpha_{N}^{i}=\frac{u^{i}}{\prod_{r \neq i}\left(u^{N}-u^{r}\right)}
$$

and hence one may calculate $\eta^{\text {rs }}$ explicitly:

$$
\begin{aligned}
\eta^{i j}(u) & =(N-1) \frac{u^{i} u^{j}}{u^{i}-u^{i}}\left[\alpha_{N}^{i}-\alpha_{N}^{j}\right], \quad r \neq s, \\
\eta^{i i}(u) & =2(N-1) \frac{\left(u^{i}\right)^{2}}{\prod_{k \neq i}\left(u^{i}-u^{k}\right)}\left[1-u^{i} \sum_{n \neq i} \frac{1}{u^{i}-u^{n}}\right] .
\end{aligned}
$$

This, togther with (12), constitutes the bi-Hamiltonian structure for the hierarchy (1), also known as the continuum Toda hierarchy.

$$
\text { If } N=2 \text { then }
$$

$$
\eta^{i j}(u)=\frac{u v}{(u-v)^{2}}\left(\begin{array}{cc}
-2 u & u+v \\
u+v & -2 v
\end{array}\right)
$$

This example also shows an interesting result of the transformation from the original to the modified variables; in the original variables the form of $g^{i j}$ is more complicated than the form of $\eta^{i j}$ while in the modified variables the complexities are interchanged.

These results depend crucially on the properties of $d_{M}$. To see this consider the flat metric 


$$
h^{i j}(u, v, w)=\left(\begin{array}{ccc}
a u^{2} & u v & u w \\
u v & a v^{2} & v w \\
u w & v w & a w^{2}
\end{array}\right)
$$

this being (12) with $N=3$ and $1-(N-M)$ replaced with an arbitrary constant $a$. We assume that this metric is invertible (so $a \neq 1,-2)$. In terms of symmetric variables $S=u+v+w, P=u v+v w+w u$ and $Q=u v w$ this takes the form

$$
h^{i j}(S, P, Q)=\left(\begin{array}{ccc}
a s^{2}+2(1-a) P & (1+a) S P+3(1-a) Q & (2+a) S Q \\
(1+a) S P+3(1-a) Q & 2(1+a) P^{2}+2(1-a) S Q & 2(2+a) P Q \\
(2+a) S Q & 2(2+a) P Q & 3(2+a) Q^{2}
\end{array}\right) .
$$

For general values of $a$ the entries are not linear in any of the variables. The metric cannot depend on $Q$ linearly as this would imply $a=-2$. For the entries to depend linearly on $Q$ would imply $a=-1$ and this corresponds to (12) with $M=1$. For the entries to depend linearly on $S$ would imply $a=0$ and this corresponds to (12) with $M=2$. Thus any requirement that the metric depends linearly on one of the symmetric variables forces the metric to take one of the above known forms. Of course this does not rule out the possibility that in some other coordinate systems the components of the metric do become linear in some variable.

\section{Rational Lax equations}

In this section the evolution equations (9) will be written in Hamiltonian form. The resulting Hamiltonian structure turns out to be degenerate, so the results of Dubrovin used in the last section to derive the bi-Hamiltonian structure cannot be used without modification. These modifications turn out to be minor and a version of lemma 4 will hold for degenerate Hamiltonian systems.

Theorem 7 (A) In terms of the variables $\tilde{u}^{i}=\log u^{i}$ the evolution equations (9) may be written in Hamiltonian form

$$
\tilde{u}_{\tau_{n}}^{i}=\sum_{j} m^{i j} \mathcal{D}\left(\frac{\delta H^{(n)}}{\delta \tilde{u}^{j}}\right)
$$

where $m^{i j}$ is the constant matrix

$$
m^{i j}=\left(\begin{array}{ccccc}
\alpha_{1} & 1 & 1 & \ldots & 1 \\
1 & \alpha_{2} & 1 & \ldots & 1 \\
1 & 1 & \alpha_{3} & \ldots & 1 \\
\vdots & \vdots & \vdots & \ddots & \vdots \\
1 & 1 & 1 & \ldots & \alpha_{N-M}
\end{array}\right)
$$


$\alpha_{i}=1-\varepsilon_{i}(N-M)$ and

$$
H^{(n)}=\int d x \sum_{\left\{r_{i}: \sum_{i=1}^{N+M} r_{i}=n\right\}}\left\{\prod_{i=1}^{N+M}\left(\begin{array}{c}
\frac{\epsilon_{i} n}{N-M} \\
r_{i}
\end{array}\right) e^{r_{i} \tilde{u}^{i}}\right\}
$$

(B) In terms of the original variables the $(g, \Gamma)$ pair

$$
\begin{aligned}
g^{i j}(u) & =m^{i j} u^{i} u^{j} \\
\Gamma_{k}^{i j}(u) & =\delta_{k}^{j} m^{i j} u^{i}
\end{aligned}
$$

define a degenerate Hamiltonian structure, satisfying the conditions of Theorem 2.

\section{Proof}

(A) In terms of the $u^{i}$ variables the system

$$
\tilde{u}_{\tau_{n}}^{i}=\sum_{j} m^{i j} \mathcal{D}\left(\frac{\delta H^{(n)}}{\delta \tilde{u}^{j}}\right)
$$

becomes

$$
u_{\tau_{n}}^{i}=\sum_{i} \alpha_{i} u^{i} \mathcal{D}\left(u^{i} \frac{\delta H^{(n)}}{\delta u^{i}}\right)+\sum_{j \neq i} u^{i} \mathcal{D}\left(u^{j} \frac{\delta H^{(n)}}{\delta u^{j}}\right) .
$$

Expanding this yields

$$
\begin{aligned}
& u_{\tau_{n}}^{i}= {\left[\frac{1}{n} \sum_{\left\{r_{i}: \sum_{i=1}^{N+M} r_{i}=n\right\}}\left[\alpha_{i} r_{i}^{2}+r_{i}\left(n-r_{i}\right)\right] \prod_{k=1}^{N+M}\left(\begin{array}{c}
\frac{\epsilon_{k} n}{N-M} \\
r_{k}
\end{array}\right)\left(u^{k}\right)^{r_{k}}\right] u_{x}^{i}+} \\
& \sum_{j \neq i}\left[\frac{1}{n} \sum_{\left\{r_{i}: \sum_{i=1}^{N+M} r_{i}=n\right\}}\left[\alpha_{i} r_{i} r_{j}+r_{j}\left(n-r_{i}\right)\right]\left(u^{j}\right)^{-1} \prod_{k=1}^{N+M}\left(\begin{array}{c}
\frac{\epsilon_{k} n}{N-M} \\
r_{k}
\end{array}\right)\left(u^{k}\right)^{r_{k}}\right] u_{x}^{j} .
\end{aligned}
$$

Using $\alpha_{i}=1-\varepsilon_{i}(N-M)$ and various binomial identities reduces this to

$$
u_{\tau_{n}}^{i}=A_{i}^{(n)} u_{x}^{i}+\sum_{j \neq i} u^{i} B_{i j}^{(n)} u_{x}^{j}
$$

where

$$
A_{i}^{(n)}=\left(\frac{\epsilon_{i} n}{N-M}-1\right) \sum_{\left\{r_{j}: \sum_{j=1}^{N+M} r_{j}=n\right\}}\left[\prod_{\substack{k=1 \\
k \neq i}}^{N+M}\left(\begin{array}{c}
\frac{\epsilon_{k} n}{N-M} \\
r_{k}
\end{array}\right)\left(u^{k}\right)^{r_{k}}\right]\left(\begin{array}{c}
\frac{\epsilon_{i} n}{N-M}-2 \\
r_{i}-1
\end{array}\right)\left(u^{i}\right)^{r_{i}}
$$


and $B_{i j}^{(n)}=$

$$
\frac{\epsilon_{j} n}{N-M} \sum_{\left\{r_{j}: \sum_{j=1}^{N+M} r_{j}=n-1\right\}}\left[\prod_{\substack{k=1 \\
k \neq i, j}}^{N+M}\left(\begin{array}{c}
\frac{\epsilon_{k} n}{N-M} \\
r_{k}
\end{array}\right)\left(u^{k}\right)^{r_{k}}\right]\left(\begin{array}{c}
\frac{\epsilon_{i} n}{N-M}-1 \\
r_{i}
\end{array}\right)\left(u^{i}\right)^{r_{i}}\left(\begin{array}{c}
\frac{\epsilon_{j} n}{N-M}-1 \\
r_{j}
\end{array}\right)\left(u^{j}\right)^{r_{j}}
$$

that is, to the equations obtained from the Lax equation (7). Hence the result.

(B) Rewritting (14) in terms of a $(g, \Gamma)$ pair yields

$$
\begin{aligned}
g^{i j}(u) & =m^{i j} u^{i} u^{j} \\
\Gamma_{k}^{i j}(u) & =\delta_{k}^{j} m^{i j} u^{i}
\end{aligned}
$$

The above argument does not show that the pair $(g, \Gamma)$ defines a Hamiltonian structure, as the corresponding bracket (1) must define a Hamiltonian structure for all functionals, not just the specific functionals used above. In order to show that this pair does define such a structure one must verify that the equations (2-6) hold. This is entirely straightforward so the details will be omitted. The degeneracy of the metric follows from the result, easily proved using elementary row and column operations, that

$$
\operatorname{det}\left(\begin{array}{cccc|ccc}
1-a & 1 & \ldots & 1 & 1 & \ldots & 1 \\
1 & 1-a & \ldots & 1 & 1 & \ldots & 1 \\
\vdots & \vdots & & \vdots & \vdots & & \vdots \\
1 & 1 & \ldots & 1-a & 1 & \ldots & 1 \\
\hline 1 & 1 & \ldots & 1 & 1+a & \ldots & 1 \\
\vdots & \vdots & & \vdots & \vdots & & \vdots \\
1 & 1 & \ldots & 1 & 1 & \ldots & 1+a
\end{array}\right)=(-1)^{N} a^{N+M-1}[a-(N-M)]
$$

where the diagonal blocks are $N \times N$ and $M \times M$ matrices. For the matrix $m_{i j} a=N-M$ (since $\alpha_{i}=1-\varepsilon_{i}(N-M)$ ) and hence $\operatorname{det}\left(g^{i j}\right)=0$. It also follows from these operations that $\operatorname{rank}\left(g^{i j}\right)=(N+M)-1$.

This also shows that this system is only mildly degenerate; the coordinate transformation that reduces $g^{i j}$ to a metric with constant entries simultaneously reduce the $\Gamma_{k}^{i j}$ to zero. For a degenerate metric this need not be the case, and some non-zero $\Gamma_{k}^{i j}$ can remain [2].

Lemma 8 Let the pair $(g, \Gamma)$ define a degenerate Hamiltonian structure. If the components of the pair $(g, \Gamma)$ in some coordinate system $x^{1}, \ldots, x^{n}$ depend linearly on the coordinate $x^{\bullet}$ then the pair

$$
\left(g+\lambda \partial_{\bullet} g, \Gamma+\lambda \partial_{\bullet} \Gamma\right)
$$

defines a degenerate Hamiltonian structure for all values of $\lambda$. Hence one obtains a degenerate bi-Hamiltonian structure. 
Proof All that is required is to show that the pair (15) satisfies the conditions of theorem 2 , given that the original $(g, \Gamma)$ pair. This is straightforward, the first two conditions being trivial. Consider, for example, condition (4) in theorem 2:

$$
\left[\left(\Gamma_{k}^{i j}+\lambda_{\bullet} \Gamma_{t}^{i j}\right)\left(g^{t k}+\lambda \partial_{\bullet} g^{t k}\right)-(k \leftrightarrow i)\right]=\left(1+\lambda \partial_{\bullet}+\frac{\lambda^{2}}{2} \partial_{\bullet}^{2}\right)\left[\left(\Gamma_{k}^{i j}-(k \leftrightarrow i)\right],\right.
$$

this following from that fact that if $g$ and $\Gamma$ depend linearly on $x^{\bullet}$ then

$$
\partial_{\bullet}^{2}(\Gamma g)=2 \partial_{\bullet} \Gamma \partial_{\bullet} g
$$

Hence if $(g, \Gamma)$ satisfies condition (田), so does (15). The remaining conditions are all quadratic in $g$ and $\Gamma$ and so the proof is identical.

One may perform a similar sequence of coordinate transformation to those in section 3. Explicitly let

$$
\begin{array}{rlr}
z^{1} & =+x^{1}, & \\
z^{i} & =+x^{i}-x^{i-1}, & \\
z^{N} & =-x^{N-1}, & \\
z^{N+1} & =+x^{N+1}, & \\
z^{i} & =+x^{i}-x^{i-1}, & \\
z^{N+M} & =-x^{N+M-1} &
\end{array}
$$

and

$$
u^{i}=e^{\frac{1}{N} x^{N}-z^{i}}, \quad i=1, \ldots, N+M .
$$

After a permutation is the labels the metric becomes (up to an overall factor)

$$
g^{i j}(x)=\left(\begin{array}{c|c|c}
+\left(\begin{array}{c}
\text { Cartan matrix } \\
\text { of } A_{N-1}
\end{array}\right) & 0 & 0 \\
\hline 0 & -\left(\begin{array}{c}
\text { Cartan matrix } \\
\text { of } A_{N+M-1}
\end{array}\right) & 0 \\
\hline 0 & 0 & -d_{M}\left(\begin{array}{ll}
1 & 1 \\
1 & 1
\end{array}\right)
\end{array}\right)
$$

This is considerable scope for the investigation of bi-Hamiltonian structures based on such block decompositions.

Having derived one Hamiltonian structure it is necessary, before the above lemma can be applied, to find a suitable coordinate system in which the metric given in theorem 7 becomes linear in one of the coordinates. This will be done only for the $M=1$ case, i.e. a 
rational Lax function with a single pole. Extending these results to an arbitrary number of pole presents certain problems, which will be discussed later.

The new variable $s^{i}$ are defined by the following expansion of the rational function $\mathcal{L}$,

$$
\begin{aligned}
\mathcal{L} & =\text { polynomial of degree }(N-1)+\frac{\text { function }}{p+\text { pole }}, \\
& =\sum_{n=0}^{N-1} p^{n} s^{N-1-n}+\frac{s^{N}}{p+s^{N+1}} .
\end{aligned}
$$

To express the $s^{i}$ as functions of the variables $u^{i}$ it is convenient to introduce the basic symmetric functions of the variables $u^{1}, \ldots, u^{N}$ :

$$
\sigma^{0}=1, \quad \sigma^{1}=\sum_{i} u^{i}, \quad \sigma^{2}=\sum_{i<j} u^{i} u^{j}, \quad \ldots \quad \sigma^{N}=\prod_{i} u^{i},
$$

SO

$$
\prod_{i=1}^{N}\left(p+u^{i}\right)=\sum_{i=0}^{N} p^{i} \sigma^{N-i} .
$$

By expanding the various expressions for $\mathcal{L}$ one obtains

$$
\begin{aligned}
s^{0} & =1, \\
s^{r} & =\sum_{n=0}^{r}(-1)^{n} \sigma^{r-n}\left(u^{N+1}\right)^{n}, \quad r=1, \ldots, N, \\
s^{N+1} & =u^{N+1} .
\end{aligned}
$$

It is in these variables that the pair $(g, \Gamma)$ will become linear in one of the variables.

Example 2 For $N=3, M=1$

$$
\begin{aligned}
\mathcal{L} & =\frac{(p+u)(p+v)}{p+w}, \\
& =p+(u+v-w)+\frac{(u-w)(v-w)}{p+w}
\end{aligned}
$$

and hence

$$
\begin{aligned}
& s^{1}=u+v-w, \\
& s^{2}=u v-w(u+v)+w^{2}, \\
& s^{3}=w
\end{aligned}
$$

in accordance with (10). 
The following result will be required in the next theorem:

Lemma 9 With the variables $s^{m}$ defined above,

$$
\frac{\partial s^{m}}{\partial u^{i}}+\frac{\partial s^{m}}{\partial u^{N+1}}=\left(u^{N+1}-u^{i}\right)\{\text { polynomial of degree } \alpha-2\}, \quad m, i=1, \ldots N .
$$

\section{Proof}

One may write $\sigma^{n}$ as $\sigma^{n}=u^{i} \tilde{\sigma}^{n-1}+\tilde{\sigma}^{n}$ so

$$
\frac{\partial \sigma^{n}}{\partial u^{i}}=\tilde{\sigma}^{n-1}
$$

Hence

$$
\begin{aligned}
\left.\frac{\partial s^{m}}{\partial u^{N+1}}\right|_{u^{N+1}=u^{i}} & =\sum(-1)^{n}\left(u^{i} \tilde{\sigma}^{n-1}+\tilde{\sigma}^{n}\right)(\alpha-n)\left(u^{i}\right)^{\alpha-n-1}, \\
& =\sum(-1)^{n+1} \tilde{\sigma}^{n-1}\left(u^{i}\right)^{\alpha-n} .
\end{aligned}
$$

So

$$
\left.\left(\frac{\partial s^{m}}{\partial u^{i}}+\frac{\partial s^{m}}{\partial u^{N+1}}\right)\right|_{u^{N+1}=u^{i}}=0 .
$$

The result now follows from the homogeneities of the functions involved.

Theorem 10 The terms of the coordinates $s^{i}$ defined above (10) the $(g, \Gamma)$ pair depend linearly on the variable $s^{N-1}$.

Proof In terms of the $u^{i}$ variables the $(g, \Gamma)$ pair are given by

$$
\begin{aligned}
g^{i j}(u) & =m^{i j} u^{i} u^{j}, \\
\Gamma_{k}^{i j}(u) & =\delta_{k}^{j} m^{i j} u^{i}
\end{aligned}
$$

where

$$
m^{i j}=\left(\begin{array}{ccccc}
2-N & 1 & \ldots & 1 & 1 \\
1 & 2-N & \ldots & 1 & 1 \\
\vdots & \vdots & \ddots & \vdots & \vdots \\
1 & 1 & \ldots & 2-N & 1 \\
1 & 1 & \ldots & 1 & N
\end{array}\right) .
$$

The components of the metric in terms of the $s^{i}$ coordinates are given by

$$
g^{i j}(s)=\frac{\partial s^{i}}{\partial u^{p}} \frac{\partial s^{j}}{\partial u^{q}} g^{p q}(u)
$$


and it follows from the symmetry of this equation that the entries will be polynomial in the new variables. It also follows from this that the degrees of the entries are

$$
\operatorname{deg} g^{i j}(s)= \begin{cases}i+j & \text { if } 1 \leq i, j \leq N \\ 1+j & \text { if } i=N+1 \text { and } j \neq N+1 \\ 1+i & \text { if } j=N+1 \text { and } i \neq N+1 \\ 2 & \text { if } i=j=N+1\end{cases}
$$

The degrees of the terms in the lower right corner of $g^{i j}(u)$ are given schematically below:

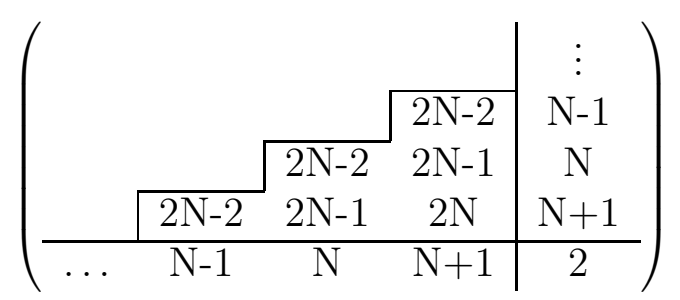

Thus there are only four terms where $g^{i j}(s)$ could possibly contain a term quadratic in $s^{N-1}$ (or six terms if $N=2$ or five terms if $N=3$, but these special cases may be disposed of by direct computation). The result will follow if it can be shown that these terms contain a factor $s^{N}$, that is if

$$
\begin{aligned}
g^{N-1, N-1}(s) & =s^{N}\{\text { polynomial of degree } N-2\}, \\
g^{N, k}(s) & =s^{N}\{\text { polynomial of degree } k\}, \quad k=N, N-1, N-2,
\end{aligned}
$$

since the polynomials cannot be quadratic in $s^{N-1}$ without violating the overall degree of the term.

From these formulae,

$$
\begin{aligned}
g^{N, \alpha}= & \sum_{i, j=1}^{N} \frac{\partial s^{N}}{\partial u^{i}} \frac{\partial s^{\alpha}}{\partial u^{j}} g^{i j}+\sum_{i=1}^{N} \frac{\partial s^{N}}{\partial u^{N+1}} \frac{\partial s^{\alpha}}{\partial u^{i}} g^{N-1, i} \\
& \sum_{i=1}^{N} \frac{\partial s^{N}}{\partial u^{i}} \frac{\partial s^{\alpha}}{\partial u^{N+1}} g^{N-1, i}+\frac{\partial s^{N}}{\partial u^{N-1}} \frac{\partial s^{\alpha}}{\partial u^{N-1}} g^{N-1, N-1} .
\end{aligned}
$$

Since

$$
s^{N}=\prod_{i=1}^{N}\left(u^{i}-u^{N+1}\right)
$$

it follows from Euler's theorem that

$$
\sum_{i=1}^{N+1} u^{i} \frac{\partial s^{N}}{\partial u^{i}}=N s^{N}
$$


and these may be used to simplify the above. After somewhat tedious calculations one obtains

$$
\frac{\partial s^{N}}{\partial u^{N-1}}=\alpha s^{N} s^{\alpha}+(1-N) s^{N}\left\{\left(u^{N+1}\right)^{2}\left[\frac{\frac{\partial s^{\alpha}}{\partial u^{i}}+\frac{\partial s^{\alpha}}{\partial u^{N+1}}}{u^{i}-u^{N+1}}\right]-u^{N+1}\left[\frac{\partial s^{\alpha}}{\partial u^{N+1}}-\frac{\partial s^{\alpha}}{\partial u^{i}}\right]\right\} .
$$

The result now follows from the above lemma.

The corresponding result for $g^{N-1, N-1}$ is similar and rest on proving, in a similar manner as above, that

$$
g^{N-1, N-1}(s)=(N-1) s^{N} \underbrace{\sum_{\substack{i \neq j \\ r \neq i, j}} \prod_{\substack{r=1 \\ r \neq i}}^{N}\left(u^{r}-u^{N+1}\right)}_{\text {symmetric polynomial in the s variables of degree }(N-2)} .
$$

These results show that in the $s^{i}$-coordinates the metric in linear in the coordinate $s^{N-1}$. The second half of the proof, showing that $\Gamma_{k}^{i j}(s)$ is also linear in $s^{N-1}$ is similar, and follows from the transformation properties of $\Gamma_{k}^{i j}$.

In what follows this second degenerately flat metric $\frac{\partial g^{i j}}{\partial s^{\bullet}}$ will be denoted by $\eta^{i j}$.

Example $3 N=2, M=1$. With these values,

$$
m^{i j}=\left(\begin{array}{lll}
0 & 1 & 1 \\
1 & 0 & 1 \\
1 & 1 & 2
\end{array}\right)
$$

and a short computation yields

$$
g^{i j}(s)=\left(\begin{array}{ccc}
2 s^{2} & s^{2}\left(s^{1}-3 s^{3}\right) & s^{3}\left(s^{1}-s^{3}\right) \\
s^{2}\left(s^{1}-3 s^{3}\right) & 2 s^{2}\left(s^{2}-s^{1} s^{3}+2\left(s^{3}\right)^{2}\right) & s^{3}\left(2 s^{2}-s^{1} s^{3}\right) \\
s^{3}\left(s^{1}-s^{3}\right) & s^{3}\left(2 s^{2}-s^{1} s^{3}\right) & 2\left(s^{3}\right)^{2}
\end{array}\right) .
$$

This is linear in $s^{1}$ and hence

$$
\begin{aligned}
\eta^{i j}(s) & =\frac{\partial g^{i j}(s)}{\partial s^{1}} \\
& =\left(\begin{array}{ccc}
0 & s^{2} & s^{3} \\
s^{2} & -2 s^{2} s^{3} & -\left(s^{3}\right)^{2} \\
s^{3} & -\left(s^{3}\right)^{2} & 0
\end{array}\right)
\end{aligned}
$$

One may easily introduce degenerate flat coordinates in which the entries of $\eta^{i j}$ are constant. These flat coordinates are: 


$$
\begin{aligned}
t^{1} & =s^{1}, \\
t^{2} & =\frac{s^{2}}{s^{3}}, \\
t^{3} & =\log \left(s^{3}\right)
\end{aligned}
$$

and in these coordinates

$$
g^{i j}(t)=\left(\begin{array}{ccc}
2 t^{2} e^{t^{3}} & -2 t^{2} e^{t^{3}} & +t^{1}-e^{t^{3}} \\
-2 t^{2} e^{t^{3}} & +2 t^{2} e^{t^{3}} & -t^{1}+e^{t^{3}} \\
+t^{1}-e^{t^{3}} & -t^{1}+e^{t^{3}} & 2
\end{array}\right) .
$$

This is linear in $t^{1}$ and hence

$$
\eta^{i j}(t)=\left(\begin{array}{ccc}
0 & 0 & +1 \\
0 & 0 & -1 \\
+1 & -1 & 0
\end{array}\right)
$$

Example $4 N=3, M=1$. With these values,

$$
m^{i j}=\left(\begin{array}{rrrr}
-1 & 1 & 1 & 1 \\
1 & -1 & 1 & 1 \\
1 & 1 & -1 & 1 \\
1 & 1 & 1 & +3
\end{array}\right)
$$

and a short computation yields the degenerate metric $g^{i j}(s)$. These components are linear in $s^{2}$ and so define a new metric

$$
\begin{aligned}
\eta^{i j}(s) & =\frac{\partial g^{i j}(s)}{\partial s^{2}}, \\
& =\left(\begin{array}{cccc}
4 & 0 & 0 & 0 \\
0 & 0 & 2 s^{3} & 2 s^{4} \\
0 & 2 s^{3} & -4 s^{3} s^{4} & -2\left(s^{4}\right)^{2} \\
0 & 2 s^{4} & -2\left(s^{4}\right)^{2} & 0
\end{array}\right) .
\end{aligned}
$$

The degenerate flat coordinates are defined by

$$
\begin{aligned}
t^{1} & =s^{1}, \\
t^{2} & =s^{2}, \\
t^{3} & =\frac{s^{3}}{s^{4}}, \\
t^{4} & =\log \left(s^{4}\right)
\end{aligned}
$$


and in these flat coordinates the original metric metric takes the form $g^{i j}(t)=$

$$
\left(\begin{array}{cccc}
-\left(t^{1}\right)^{2}+4 t^{2} & +6 t^{3} e^{t^{4}} & -6 t^{3} e^{t^{4}} & t^{1}-2 e^{t^{4}} \\
+6 t^{3} e^{t^{4}} & +4 t^{1} t^{3} e^{t^{4}}-8 t^{3} e^{2 t^{4}} & -4 t^{1} t^{3} e^{t^{4}}+8 t^{3} e^{2 t^{4}} & +2 t^{2}-2 t^{1} e^{t^{4}}+2 e^{2 t^{4}} \\
-6 t^{3} e^{t^{4}} & -4 t^{1} t^{3} e^{t^{4}}+8 t^{3} e^{2 t^{4}} & +4 t^{1} t^{3} e^{t^{4}}-8 t^{3} e^{2 t^{4}} & -2 t^{2}+2 t^{1} e^{t^{4}}-2 e^{2 t^{4}} \\
t^{1}-2 e^{t^{4}} & +2 t^{2}-2 t^{1} e^{t^{4}}+2 e^{2 t^{4}} & -2 t^{2}+2 t^{1} e^{t^{4}}-2 e^{2 t^{4}} & 3
\end{array}\right) .
$$

The entries are linear in $t^{2}$ and hence one obtains the second metric

$$
\eta^{i j}(t)=\left(\begin{array}{rrrr}
4 & 0 & 0 & 0 \\
0 & 0 & 0 & 2 \\
0 & 0 & 0 & -2 \\
0 & 2 & -2 & 0
\end{array}\right)
$$

\section{Degenerate Frobenius manifolds}

The natural geometric setting in which to understand the bi-Hamiltonian structure of hydrodynamic systems is the Frobenius manifold [5]. One way to defined such manifolds is to construct a function $F\left(t^{1}, \ldots, t^{n}\right)$ such that the associated functions

$$
c_{i j k}=\frac{\partial^{3} F}{\partial t^{i} \partial t^{j} \partial t^{k}}
$$

satisfy the following conditions:

- the matrix $\eta_{i j}=c_{1 i j}$ is constant and non-degenerate. This together with the inverse matrix $\eta^{i j}$ are used to raise and lower indices. On such a manifold one may interpret $\eta_{i j}$ as a flat metric;

- the functions $c_{j k}^{i}=\eta^{i r} c_{r j k}$ defined an associative commutative algebra with a unity element. This defines a Frobenius algebra on each tangent space $T^{t} \mathcal{M}$. This multiplication will be denoted by $u \cdot v$;

- the functions $F$ satisfies a quasi-homogeneity condition, which may be expressed as

$$
\mathcal{L}_{E} F=d_{F} F+\{\text { quadratic terms }\},
$$

where $E$ is a vector fields known as the Euler vector field.

These conditions constitute the Witten-Dijkgraaf-Verlinde-Verlinde (or WDVV) equations. On such a manifold one may introduce a second flat metric defined by

$$
g^{i j}=E\left(d t^{i} \cdot d t^{j}\right) .
$$


This metric, together with the original metric $\eta^{i j}$ define a flat pencil (i.e. $\eta^{i j}+\lambda g^{i j}$ is flat for all values of $\lambda$. Thus one automatically obtains a bi-Hamiltonian structure from a Frobenius manifold. The corresponding Hamiltonians are defined recursively by the formula

$$
\frac{\partial^{2} h^{(n)}}{\partial t^{i} \partial t^{j}}=c_{i j}^{k} \frac{\partial h^{(n-1)}}{\partial t^{k}} .
$$

the integrability conditions for this systems are automatically satisfied when the $c_{i j}^{k}$ are defined as above.

One basic assumption in this definition is that the metric $\eta_{i j}$ is non-degenerate, and it follows from this that the bi-Hamiltonian structures are also non-degenerate. Thus the degenerate bi-Hamiltonian structures obtained in the preceding section cannot be obtained from this construction. However, one may formulate the new notion of a degenerate Frobenius manifold in which the corresponding bi-Hamiltonian structures are degenerate.

Rather than develop the theory of degenerate Frobenius manifolds in full generality an extended example will be given here based on the study of the hydrodynamic system

$$
\begin{aligned}
u_{\tau} & =u\left(v_{x}-w_{x}\right), \\
v_{\tau} & =v\left(u_{x}-w_{x}\right), \\
w_{\tau} & =w\left(u_{x}+v_{x}-2 w_{x}\right)
\end{aligned}
$$

obtained from the rational Lax function

$$
\mathcal{L}=\frac{(p+u)(p+v)}{p+w} .
$$

The bi-Hamiltonian structure of this system has already been derived in example 3 in section 4 . To recapitulate, in the flat coordinates given by

$$
\begin{aligned}
t^{1} & =u+v-w, \\
t^{2} & =\frac{(u-w)(v-w)}{w}, \\
t^{3} & =\log w
\end{aligned}
$$

the degenerate metrics which give rise to the degenerate bi-Hamiltonian structures are:

$$
\begin{aligned}
\eta^{i j}(t) & =\left(\begin{array}{ccc}
0 & 0 & +1 \\
0 & 0 & -1 \\
+1 & -1 & 0
\end{array}\right), \\
g^{i j}(t) & =\left(\begin{array}{ccc}
2 t^{2} e^{t^{3}} & -2 t^{2} e^{t^{3}} & +t^{1}-e^{t^{3}} \\
-2 t^{2} e^{t^{3}} & +2 t^{2} e^{t^{3}} & -t^{1}+e^{t^{3}} \\
+t^{1}-e^{t^{3}} & -t^{1}+e^{t^{3}} & 2
\end{array}\right) .
\end{aligned}
$$


The first few Hamiltonian densities (suitably normalised) are given by the formula (10), and in the flat coordinates these become

$$
\begin{aligned}
h^{(1)} & =t^{1} \\
h^{(2)} & =\frac{1}{2}\left[\left(t^{1}\right)^{2}+2 t^{2} e^{t^{3}}\right] \\
h^{(3)} & =\frac{1}{6}\left[\left(t^{1}\right)^{3}+6 t^{1} t^{2} e^{t^{3}}-3 t^{2} e^{2 t^{3}}\right], \\
h^{(4)} & =\frac{1}{24}\left[\left(t^{1}\right)^{4}+12\left(t^{1}\right)^{2} t^{2} e^{t^{3}}+6\left(t^{2}\right)^{2} e^{2 t^{3}}-12 t^{1} t^{2} e^{2 t^{3}}+4 t^{2} e^{3 t^{3}}\right] .
\end{aligned}
$$

From these and the recursion equation (21) one may reconstruct the structure functions $c_{j k}^{i}$ and verify that they form an commutative and associative algebra with a unity element. Explicity the structure constants are given by $c_{1 j}^{i}=\delta_{j}^{i}$ and

$$
\left(\begin{array}{ccc}
c_{22}^{1} & c_{22}^{2} & c_{22}^{3} \\
c_{23}^{1} & c_{23}^{2} & c_{23}^{3} \\
c_{33}^{1} & c_{33}^{2} & c_{33}^{3}
\end{array}\right)=\left(\begin{array}{ccc}
0 & 1 & -1 / t^{2} \\
+e^{t^{3}} & -e^{t^{3}} & 0 \\
+t^{2} e^{t^{3}} & -t^{2} e^{t^{3}} & -e^{t^{3}}
\end{array}\right) .
$$

From these structure functions one may raise an index using $\eta^{i j}$ and determine the Euler vector field from equation (20). For this example this vector field is

$$
E=t^{1} \frac{\partial}{\partial t^{1}}+t^{2} \frac{\partial}{\partial t^{2}}+\frac{\partial}{\partial t^{3}}
$$

In addition, the structure functions satisfy the relations

$$
\frac{\partial c_{j k}^{r}}{\partial t^{i}}-\frac{\partial c_{i k}^{r}}{\partial t^{k}}=0
$$

and this, together with the symmetry $c_{i j}^{k}=c_{j i}^{k}$ enables one to write the them as

$$
c_{j k}^{i}=\frac{\partial^{2} f^{i}}{\partial t^{j} \partial t^{k}}
$$

for some set of functions $f^{i}$. For the above structure constants these turn out to be (up to linear terms),

$$
\begin{aligned}
f^{1} & =\frac{1}{2}\left(t^{1}\right)^{2}+t^{2} e^{t^{3}} \\
f^{2} & =\frac{1}{2}\left(t^{2}\right)^{2}+t^{1} t^{2}-t^{2} e^{t^{3}} \\
f^{3} & =t^{1} t^{3}-t^{2} \log t^{2}-e^{t^{3}}
\end{aligned}
$$

At this stage one normally lowers the $i$ index and use another symmetry to write $c_{i j k}$ as the third derivative of some function $F$. This, however, assumes that the metric $\eta^{i j}$ is 
invertible, which, for the metric given by (22) is not the case. However one may write the $f^{i}$ as

$$
f^{i}(t)=\eta^{i j} \frac{\partial F}{\partial t^{j}}+h^{i}\left(t^{1}+t^{2}\right) .
$$

Since the matrix $\eta^{i j}$ is of rank 2 it follows it has a non-trivial kernel, so there exists a nonzero vector $\zeta_{i}$ such that $\eta^{i j} \zeta_{j}=0$, and the functions $h^{i}$ are functions of the combination $\zeta_{i} t^{i}$, which in this example is just $t^{1}+t^{2}$. These functions $h^{i}$ satisfy the single constraint $h^{1}+h^{2}=1 / 2\left(t^{1}+t^{2}\right)^{2}$. To obtain the above structure functions one possible such $F$ is

$$
F=\frac{1}{2}\left(t^{1}\right)^{2} t^{3}+t^{2} e^{t^{3}}+\frac{1}{2}\left(t^{2}\right)^{2} \log t^{2},
$$

and

$$
\begin{aligned}
h^{1} & =0, \\
h^{2} & =\frac{1}{2}\left(t^{1}+t^{2}\right)^{2}, \\
h^{3} & =0 .
\end{aligned}
$$

and this satisfies the homogeneity condition (19) with $d_{F}=2$. There is much freedom in these functions. One may transform, for arbitrary constant $k, F$

$$
F \longrightarrow F+k t^{3}\left(t^{1}+t^{2}\right)^{2}
$$

and the homogeneity property is unchanged. This induced a change in the functions $h^{i}$ but leaves unchanged the structure functions defining the Frobenius algebra.

From this extended example one may distil the basic properties of a degenerate Frobenius manifold. One starts with a basic function $F\left(t^{i}\right)$ satisfying some homogeneity condition and degenerate metric $\eta^{i j}$, the entries of which are constant in the $t^{i}$-coordinates. The metric is not related to the third derivatives of $F$, as for non-degenerate Frobenius manifolds. The structure functions, which form a Frobenius algebra with a degenerate inner product, are defined by

$$
c_{j k}^{i}=\eta^{i r} \partial_{r} \partial_{j} \partial_{k} F+\partial_{j} \partial_{k} h^{i},
$$

where the functions $h^{i}$ are functions which depend on the kernel of the degenerate matrix $\eta^{i j}$. Thus for degenerate Frobenius manifolds one has a set of extra functions related to the fact that the matrix $\eta^{i j}$ is not of maximal rank. The associativity conditions results in a complicated set over-determined partial differential equations for $F$, the degenerate analogue of the WDVV equations. One avenue for future research is to develop the concept of a degenerate Frobenius manifold more axiomatically. 
Example 5 For $N=3, M=1$ the metrics $g^{i j}(t)$ and $\eta^{i j}(t)$ have been calculated in example 4. One may repeat the calculations above and obtain

- Euler vector field:

$$
E=\frac{t^{1}}{2} \frac{\partial}{\partial t^{1}}+t^{2} \frac{\partial}{\partial t^{2}}+t^{3} \frac{\partial}{\partial t^{3}}+\frac{1}{2} \frac{\partial}{\partial t^{4}}
$$

- Prepotential F :

$$
F=\frac{1}{8}\left(t^{1}\right)^{2} t^{2}+\frac{1}{4}\left(t^{2}\right)^{3}-\frac{1}{192}\left(t^{1}\right)^{4}+\frac{1}{2} t^{1} t^{3} e^{t^{4}}-\frac{1}{4} t^{3} e^{2 t^{4}}-\frac{1}{4}\left(t^{3}\right)^{2} \log t^{3}
$$

- associated non-zero potentials $h^{i}$ :

$$
h^{3}=\frac{1}{2}\left(t^{2}+t^{3}\right)^{2}
$$

From these, and the constant matrix $\eta^{i j}$ given by (18), one may construct a degenerate Frobenius algebra with structure functions given by equation (24) and second degenerate flat metric given by (20).

In section 4 the bi-Hamiltonian structures were shown to exist for arbitrary $N$ but $M=1$. It is clear that the ideas will generalize to arbitrary $M$, and hence to degenerate Frobenius manifolds for arbitrary $N$ and $M$. The following example is for $N=3, M=2$.

Example 6 For $N=3, M=2$ the flat coordinates are defined by the expansion

$$
\mathcal{L}=p+t^{1}+\frac{t^{2} e^{t^{4}}}{p+e^{t^{4}}}+\frac{t^{3} e^{t^{5}}}{p+e^{t^{5}}}
$$

In these coordinates

$$
\eta^{i j}(t)=\left(\begin{array}{rrrrr}
0 & 0 & 0 & 1 & 1 \\
0 & 0 & 0 & -1 & 0 \\
0 & 0 & 0 & 0 & -1 \\
1 & -1 & 0 & 0 & 0 \\
1 & 0 & -1 & 0 & 0
\end{array}\right)
$$

and the Frobenius data is:

- Euler vector field:

$$
E=t^{1} \frac{\partial}{\partial t^{1}}+t^{2} \frac{\partial}{\partial t^{2}}+t^{3} \frac{\partial}{\partial t^{3}}+\frac{\partial}{\partial t^{4}}+\frac{\partial}{\partial t^{5}}
$$


- Prepotential F :

$$
\begin{aligned}
F & =+t^{2} e^{t^{4}}+t^{3} e^{t^{5}}+\frac{1}{2} t^{2} t^{3} \log \left(e^{t^{4}}-e^{t^{5}}\right)^{2}+\frac{1}{2}\left(\left(t^{2}\right)^{2} \log t^{2}+\left(t^{3}\right)^{2} \log t^{3}\right) \\
& =-\frac{1}{2} t^{2} t^{4}\left(2 t^{1}+t^{2}+2 t^{3}\right)-\frac{1}{2} t^{3} t^{5}\left(2 t^{1}+2 t^{2}+t^{3}\right)
\end{aligned}
$$

- associated non-zero potentials $h^{i}$ :

$$
h^{1}=\frac{1}{2}\left(t^{1}+t^{2}+t^{3}\right)^{2} .
$$

From this data the Frobenius algebra structure functions given by equation (24) and the second degenerate flat metric given by (20).

The form of these results suggest the following:

Conjecture 1 The metric given in Theorem 7 is linear in the coordinate $s^{N-M}$, where the coordinates $s^{i}$ are defined in terms of the expansion of the rational Lax function

$$
\mathcal{L}=p^{N-M}+s^{1} p^{N-M-1}+\ldots+s^{N-M}+\frac{s^{N-M+1}}{p+s^{N+1}}+\ldots+\frac{s^{N}}{p+s^{N+M}} .
$$

Moreover, there exist flat coordinates $t^{i}$ such that the variables $s^{i}$ are polynomial functions of the variables $t^{1}, \ldots, t^{N}, e^{t^{N+1}}, \ldots e^{t^{M}}$, and in which the entries $\eta^{i j}(t)$ are all constants.

One would hope to be able to modify the results of [6] to prove this conjecture; the vanishing of the determinants of the metrics means that the results cannot be used directly. One should be able to modify the Gauss-Manin equations for the flat coordinates to include these degenerate examples.

\section{Comments}

One notable difference between the bi-Hamiltonian structure of the hierarchies consider here, these being multi-component generalizations of Toda and Benney hierarchies [4], and the bi-Hamiltonian structures of dispersionless KP-type hierarchies is the degeneracy of the structures. The dispersionless KP-type hydrodynamic systems involve rational such as (see, for example, those in [9])

$$
\mathcal{L}=\frac{1}{2} p^{2}+S(x, t)+\frac{P(x, t)}{p-Q(x, t)}
$$

and Lax equation similar to equation (7), but with Poisson bracket 


$$
\{f, g\}_{P B}=\left(\partial_{p} f \partial_{x} g-\partial_{x} f \partial_{p} g\right) .
$$

The bi-Hamiltonian structure of these equations are not degenerate [5, 9]. These rational Lax functions may be considered as a reduction of an infinite component Lax function $\mathcal{L}=\sum_{i=-\infty}^{N} s^{i} p^{i}$ and it may be of interest to see how constraining the resulting Hamiltonian structures results in the degenerate structures studied here.

The existence of a non-trivial Casimir for these systems is of interest. One possible reduction of these systems is to restrict the dynamics to the surface given by

$$
\mathcal{C}=\text { constant }
$$

for example the $(N=2, M=1)$ system

$$
\begin{aligned}
u_{\tau} & =u\left(v_{x}-w_{x}\right), \\
v_{\tau} & =v\left(u_{x}-w_{x}\right), \\
w_{\tau} & =w\left(u_{x}+v_{x}-2 w_{x}\right)
\end{aligned}
$$

when restricted to the surface $w=u v$ results in the system

$$
\begin{aligned}
& u_{\tau}=u\left[(1-u) v_{x}-v u_{x}\right], \\
& v_{\tau}=v\left[(1-v) u_{x}-u v_{x}\right] .
\end{aligned}
$$

How the Hamiltonian structure behaves under such a constraint is unknown. For nondegenerate Hamiltonian structures one may use the result of Ferapontov [10], though this work would need to be generalised to include degenerate Hamiltonian structure such as those considered here. More generally, one may restrict the above system to the surface $w=u v f(x)$ for some arbitrary function $f(x)$ (i.e. $\mathcal{C}=f^{-1}$.). This results in the system

$$
\begin{aligned}
& u_{\tau}=u\left[(1-u) v_{x}-v u_{x}\right]-u^{2} v f^{\prime}(x), \\
& v_{\tau}=v\left[(1-v) u_{x}-u v_{x}\right]-v^{2} u f^{\prime}(x),
\end{aligned}
$$

an example of inhomogeneous hydrodynamic system with specific $x$-dependence. It may also be possible to obtain the Hamiltonian structure of these systems [11].

The idea of a degenerate Frobenius manifold requires further elucidation. One complicating factor is that for a degenerate structure the transformation which reduces the components of the metric to constants will not, in general, reduce all of the components $\Gamma_{k}^{i j}$ to zero [2]. The systems in this paper are special in this respect since in flat coordinates the components of $\Gamma_{k}^{i j}$ are automatically zero, which is not the generic suituation; the systems here are doubly degenerate. 
Finally, this paper has only dealt with dispersionless systems. For polynomial Lax equations one has discrete counterparts, the simplest example being the Toda Lattice

$$
\begin{aligned}
& S_{n, \tau}=P_{n}-P_{n+1}, \\
& P_{n, \tau}=P_{n}\left(S_{n-1}-S_{n}\right)
\end{aligned}
$$

which reduces to (8) in the continuum limit; the lattice variable becoming the continuous variable $x$. The bi-Hamiltonian structure of such systems have been studied in [0]. Indeed, the structures obtained here could also be derived by taking certain limits of those structures, if they where known explicitly for arbitrary $M$ and $N$. How to extend these results to rational discrete systems is unclear. One approach would be to use the ideas in [12], which deals with the interpretation of the inverse operator $\left(e^{\partial}+u\right)^{-1}$, or the ideas of

13] where one would consider term-by-term deformation of the underlying dispersionless system.

\section{Acknowledgments}

I would like to thank Prof. A.P. Fordy for informing me that the result of Dubrovin [5] was a special case of a result of Magri [8] .

\section{References}

[1] Dubrovin, B. and Novikov, S.P. Hydrodynamics of weakly deformed soliton lattices. Differential geometry and Hamiltonian theory, Russ. Math. Surv. 44:6 (1989) 35-124.

[2] Grinberg, N.I. On Poisson brackets of hydrodynamic type with a degenerate metric, Russ. Math. Surv. 40:4 (1985) 231-232.

[3] Dorfmann, I. Dirac Structures and Integrability of Nonlinear Evolution Equations, Wiley (1993).

[4] Fairlie, D.B. and Strachan, I.A.B. The algebraic and Hamiltonian structure of the dispersionless Benney and Toda hierarchies, Inverse problems 12 (1996) 885-908.

[5] Dubrovin, B. Geometry of 2D topological field theories in Integrable Systems and Quantum Groups, Montecalini, terms, 1993. Editors: M. Francaviglia and S. Greco. Springer lecture notes in mathematics, 1620 (1996), 120-3348.

[6] Dubrovin, B. and Zhang, Y. Extended affine Weyl groups and Frobenius manifolds, Compositio Math., 1997. 
[7] Kupershmidt, B.A., Discrete Lax Equations and Differential Difference Calculus, Astérisque 123 1-212.

[8] Magri, F. A simple model of the integrable Hamiltonian equation, J. Math. Phys. 19 (1978) 1156-1162.

[9] Aoyama, S and Kodama, Y. Topological Landau-Ginzburg theory with a rational potential and the dispersionless KP hierarchy, Commum. Math. Phys. 182 (1996) $185-219$.

[10] Ferapontov, E.V. Dirac reduction of the operator $\delta^{i j} d / d x$ to a submanifold with flat normal connection, Func. Ann. Appl. 26 (1992) 83-86.

[11] Polyak, M.V. One-dimensional Hamiltonian systems of hydrodynamic type with an explicit dependence on the spacial variable, Russ. Math. Surv. 42.3 (1987) 229-230.

[12] Enriques, B, Orlov, A. Yu and Rubtsov, V.N., Dispersionful analogues of Benney's equations and $N$-wave systems, Inverse Problems 12 241-50.

[13] Eguchi, T., Yamada Y. and Yang S.K., On the genus expansion in the topological string theory, Rev. Math. Phys. 7 (1995) 279. 